\title{
Confiabilidade da Medida de Espessuras Musculares Pela Ultrassonografia
}

\author{
Reliability of Muscle Thickness Measurements Using Ultrasound
}



Artigo Original

Paulo Sergio Chagas Gomes ${ }^{1,2}$ Cláudia de Mello Meirelles ${ }^{1,3}$ Sandra Pereira Leite ${ }^{4}$

Carlos Antonio Barbosa

Montenegro ${ }^{5}$

1. Laboratório Crossbridges da Universidade Gama Filho - Rio de Janeiro.

2. Programa de Pós-Graduação em Educação Física da Universidade

Gama Filho - Rio de Janeiro.

3. Escola de Educação Física do

Exército - Rio de Janeiro.

4. Rede Labs D'Or - Rio de Janeiro.

5. Curso de Medicina da

Universidade Gama Filho e

Ultrassonografia Botafogo - Rio de Janeiro.

Endereço para correspondência Departamento de Educação Física, Universidade Gama Filho.

Rua Manoel Vitorino, 625.

20748-900 - Rio de Janeiro, RJ, Brasil

Tel./fax: (21) 2599-7138

E-mail: crossbridges@ugf.br

\begin{abstract}
RESUMO
Objetivo: Determinar a confiabilidade das medidas de espessuras dos músculos flexores e extensores do cotovelo e joelho pela ultrassonografia (US), quantificando o erro típico associado a essas medidas (ETM). Métodos: A confiabilidade (duas medidas interdias) foi determinada em 15 voluntários aparentemente saudáveis (oito mulheres, $33,9 \pm 11,4$ anos, $76 \pm 21 \mathrm{~kg}, 170 \pm 10 \mathrm{~cm}$ ). As imagens da musculatura flexora (FC) e extensora do cotovelo (EC) e flexora (FJ) e extensora do joelho (EJ) foram obtidas pela US bidimensional no modo $\mathrm{B}$, utilizando transdutor de $7,5 \mathrm{MHz}$. As espessuras do tecido muscular compreendidas entre as interfaces com o osso e com o tecido adiposo foram medidas em sítios anatômicos identificados e registrados para ser repetidos na segunda medida. Resultados: A ANOVA não identificou diferenças significativas entre as medidas repetidas. Os coeficientes de correlação intraclasse foram $\mathrm{FC}=0,970, \mathrm{EC}=0,971$, FJ $=0,555$ e $E J=0,929$ ( $P<0,05$ para todos). Os coeficientes de variação foram de 3,9\%, $6,1 \%, 6,6 \%$ e 4,6\%, e os ETM $1,3 \mathrm{~mm}, 1,6 \mathrm{~mm}, 4,9 \mathrm{~mm}$ e 1,9mm, respectivamente, para espessuras de FC, EC, FJ e EJ. A análise dos gráficos de Bland-Altman apontou que as variáveis não apresentaram erro heterocedástico. Conclusão: As medidas de espessuras musculares de FC, EC e EJ apresentaram alta confiabilidade, possibilitando seu uso para intervenções como programas de dieta, treinamento físico ou reabilitação. No entanto, o uso da medida de FJ não deve ser recomendada, pois mostrou baixa confiabilidade e alto ETM.
\end{abstract}

Palavras-chave: hipertrofia, reprodutibilidade, músculo esquelético.

\begin{abstract}
Purpose: To determine the reliability of muscle thickness measurements of elbow and knee flexors and extensors using ultrasound, and to quantify the typical error associated to the measurements (TEM). Methods: The test-retest reliability was determined in 15 apparently healthy volunteers (8 women, $34 \pm 11$ years, 76 $\pm 21 \mathrm{~kg}, 170 \pm 10 \mathrm{~cm}$ ). The images of elbow flexors (EF) and extensors (EE) and knee flexors (KF) and extensors (KE) were obtained using a two dimensional mode B ultrasound instrument with a 7.5 MHz transducer Muscle thickness between the adipose tissue and bone interfaces were measured at anatomical landmarks previously identified and recorded to assure the exact site for the retest. Results: ANOVA did not identify any significant differences between the repeated measurements. Intraclass correlation coefficients (ICC) of each pair of measure were $\mathrm{EF}=0.970, \mathrm{EE}=0.971, \mathrm{KF}=0.555$ e KE $=0.929(\mathrm{P}<0.05$ for all). The coefficients of variation were $3.9 \%, 6.1 \%, 6.6 \%$ e $4.6 \%$, and TEM $1.3 \mathrm{~mm}, 1.6 \mathrm{~mm}, 4.9 \mathrm{~mm}$ e $1.9 \mathrm{~mm}$, respectively, for the EF, EE, KF and KE thickness. Analysis of the Bland-Altman plot charts indicated that the variables did not present heterocedastic errors. Conclusion: $\mathrm{EF}$, EE and KE muscle thickness measurements using the current method were highly reliable and can be used to measure and monitor changes in diet, physical training and rehabilitation programs. Nevertheless, KF measure should not be recommended since it presented low reliability and high TEM.
\end{abstract}

Keywords: hypertrophy, reproducibility, skeletal muscle.

\section{INTRODUÇÃO}

A ultrassonografia (US) tem se mostrado um método válido(1-3) para a determinação de dimensões musculares e, entre os demais métodos indiretos para medida do tecido muscular por imagem (ressonância nuclear magnética - RNM e tomografia computadorizada - TC), a US representa o de menor custo e maior inocuidade, uma vez que não expõe o avaliado à radiação, como ocorre com a TC.

A US pode ser empregada na monitoração das modificações na massa muscular decorrentes dos efeitos de programas de dieta e/ou exercícios voltados à promoção da saúde ou à reabilitação osteomioarticular.

Starkey et al. ${ }^{(4)}$ utilizaram a US para observar os efeitos de 14 semanas de treinamento contrarresistência sobre as espessuras musculares e observaram aumentos estatisticamente significativos, mas de pequena magnitude, ou seja, de aproximadamente $1 \mathrm{~mm}$ na face ântero-medial de extensores do joelho até aproximadamente 3,4mm em flexores do joelho. Como as modificações inerentes ao tratamento podem ser pequenas, a precisão do método de obtenção da medida precisa ser muito alta, podendo assim ser capaz de identificar as pequenas modificações inerentes à intervenção e distingui-las do erro na obtenção das medidas.

A tabela 1 mostra o resultado de estudos( ${ }^{(4-6)}$ que empregaram a US para obter medidas musculares antes e após intervenções de treinamento ou destreinamento, inclusive aqueles que aplicaram as medidas de espessuras em equações que permitiram a estimativa de área de seção transversa muscular ${ }^{(7)}$. 
Tabela 1. Estudos que investigaram o efeito do treinamento contrarresistência (TCR), da eletromioestimulação (EMS) ou do destreinamento (DET) sobre as espessuras ou área de seção transversa musculares pela ultrassonografia (média \pm desvio padrão)

\begin{tabular}{|c|c|c|c|c|c|}
\hline \multirow{2}{*}{ Fonte } & \multirow{2}{*}{$n$} & \multirow{2}{*}{ Estímulo } & \multirow{2}{*}{ Sítio(s) } & \multicolumn{2}{|c|}{ Espessura (mm) } \\
\hline & & & & Pré-TCR & Pós-TCR \\
\hline 4 & $\begin{array}{c}18 \\
\mathrm{HeM} \\
\mathrm{NT}\end{array}$ & $\begin{array}{c}\text { TCR } \\
1 \text { série } \\
\text { 8-12 reps } \\
3 x / \text { SEM } \\
14 \text { SEM }\end{array}$ & $\begin{array}{l}\text { EJ } 40 \% \\
\text { EJ } 60 \% \\
\text { FJ } 40 \% \\
\text { FJ } 60 \% \\
\text { EJ AM }\end{array}$ & $\begin{array}{c}52,0 \pm 6,6 \\
40,1 \pm 6,8 \\
60,9 \pm 11,3 \\
62,4 \pm 9,2 \\
41,0 \pm 7,3\end{array}$ & $\begin{array}{c}53,1 \pm 6,6 \\
41,1 \pm 6,8 \\
64,3 \pm 10,7^{*} \\
65,1 \pm 9,1^{*} \\
42,1 \pm 7,3\end{array}$ \\
\hline 4 & $\begin{array}{c}20 \\
\text { HeM } \\
\text { NT }\end{array}$ & $\begin{array}{c}\text { TCR } \\
3 \text { séries } \\
\text { 8-12 reps } \\
3 x / \text { SEM } \\
14 \text { SEM }\end{array}$ & $\begin{array}{l}\text { EJ } 40 \% \\
\text { EJ } 60 \% \\
\text { FJ } 40 \% \\
\text { FJ } 60 \% \\
\text { EJ AM }\end{array}$ & $\begin{array}{l}49,3 \pm 8,0 \\
38,0 \pm 7,5 \\
56,1 \pm 7,0 \\
58,9 \pm 7,0 \\
38,8 \pm 7,2\end{array}$ & $\begin{array}{l}50,3 \pm 7,3 \\
39,1 \pm 7,3 \\
59,1 \pm 7,8 \\
61,3 \pm 6,9^{*} \\
39,8 \pm 6,5^{*}\end{array}$ \\
\hline 5 & $\begin{array}{c}16 \\
\text { Hjovens } \\
\text { NT }\end{array}$ & $\begin{array}{c}\text { TCR } \\
\text { 3-4 séries; } \\
\text { 6-12 reps } \\
\text { 30-60\% 1RM } \\
\text { 3x/SEM; } 13 \text { SEM }\end{array}$ & $V L$ & $23,0 \pm 4,2$ & $24,6 \pm 3,6^{*}$ \\
\hline 6 & $\begin{array}{l}14 \mathrm{He} 15 \mathrm{M} \\
\text { jovens }\end{array}$ & $\begin{array}{c}\text { TCR isocinético } \\
\text { Extensão joelho } \\
60^{\circ} \cdot 5-1 \\
3 x / S E M \\
5 \text { SEM }\end{array}$ & $\mathrm{VL}$ & $21,9 \pm 3,2$ & $22,0 \pm 4,3$ \\
\hline Fonte & $\mathrm{n}$ & Estímulo & Sítio(s) & \multicolumn{2}{|c|}{ Área de seção transversa $\left(\mathrm{cm}^{2}\right)$} \\
\hline 7 & $\begin{array}{c}9 \text { Hjovens } \\
\text { NT }\end{array}$ & $\begin{array}{c}\text { EMS } \\
18 \text { min/40 } \\
\text { contrações } \\
4 \times / S E M \\
8 \text { SEM }\end{array}$ & $V L+V I+V M$ & $71,8 \pm 3,7$ & $76,7 \pm 3,4^{*}$ \\
\hline 7 & $\begin{array}{l}9 \text { H jovens } \\
\text { T/EMS }\end{array}$ & $\begin{array}{c}\text { DET } \\
4 \text { SEM }\end{array}$ & $\mathrm{VL}+\mathrm{VI}+\mathrm{VM}$ & $76,7 \pm 3,4$ & $74,0 \pm 2,9^{*}$ \\
\hline
\end{tabular}

H: homens; M: mulheres; T: treinados; NT: não treinados; ${ }^{*} \mathrm{P}<0,05$ em relação ao valor anterior; SEM: semanas; reps: repetições; 1 RM: 1 repetiçăo máxima; EJ: extensor do joelho; FJ: flexor do joelho; AM: face ântero-media de EJ; 40 \% e 60 \%: pontos anatômicos situados a 40\% e 60\% da distância entre o epicôndilo lateral da tíbia e o grande trocânter, a partir deste último; VL: vasto lateral; VM: vasto medial; Vl: vasto intermédio
A confiabilidade da medida parece estar sujeita a diversas variáveis, tais como a resolução do equipamento, a identificação precisa dos sítios anatômicos e a experiência do ultrassonografista. Também variáveis individuais, como o adequado relaxamento da musculatura a ser monitorada e o tempo transcorrido desde a última sessão de exercícios físicos e a tomada da medida, podem afetar a qualidade da medida.

Ishida et al. ${ }^{(8)}$ consideraram a US um método confiável para medidas de espessuras musculares entre as interfaces com o osso e com o tecido adiposo. Outros resultados estão disponíveis, porém, são restritos, uma vez que a maioria não relata informações relevantes tais como o ETM ${ }^{(5,8,9-11)}$ e o $\mathrm{CCl}^{(10)}$, enquanto outros relatam os $\mathrm{CCl}$, porém, não especificam a que grupos musculares se referem ${ }^{(6,12)}$. A tabela 2 mostra que os resultados são diversos, podendo se referir a espessuras, à área de seção transversa ou ao volume de músculos ou grupamentos musculares.

Em investigações científicas ou acompanhamentos clínicos é de suma importância identificar os efeitos da intervenção sobre as dimensões musculares e conhecer o ruído na obtenção das medidas. Dessa forma, o objetivo deste estudo foi determinar a confiabilidade das medidas das espessuras dos músculos flexores e extensores do cotovelo e joelho, quantificando o erro típico associado a essas medidas.

\section{MÉTODOS}

\section{Amostra}

Participaram 15 sujeitos (oito mulheres) aparentemente saudáveis $(33,9 \pm 11,4$ anos, $76 \pm 21 \mathrm{~kg}, 170 \pm 10 \mathrm{~cm})$. Todos os voluntários foram esclarecidos sobre os procedimentos experimentais e a inexistência de riscos associados ao estudo, conforme determinações institucionais e a Resolução 96/196 do Conselho Nacional de Saúde.

Tabela 2. Estudos que investigaram a confiabilidade da estimativa de volumes (VOL) ou medida de espessuras (ESP) e área de seção transversa (AST) musculares pela ultrassonografia (média \pm desvio padrão)

\begin{tabular}{|c|c|c|c|c|c|c|c|}
\hline Fonte & $\mathrm{n}$ & Sítio(s) & VOL $\left(\mathrm{cm}^{3}\right)$ & $\mathrm{CCl}(\mathrm{R})$ & CV (\%) & $\mathrm{CR}\left(\mathrm{cm}^{3}\right)$ & $\operatorname{ETM}\left(\mathrm{cm}^{3}\right)$ \\
\hline 1 & $6 \mathrm{He} \mathrm{M}$ adultos & TA & $133 \pm 21$ & 0,984 & NR & NR & NR \\
\hline Fonte & $\mathrm{n}$ & Sítio(s) & $\mathrm{ESP}(\mathrm{mm})$ & $\mathrm{CCl}(\mathrm{R})$ & CV (\%) & $\mathrm{CR}(\mathrm{mm})$ & $\operatorname{ETM}(\mathrm{mm})$ \\
\hline 2 & $\begin{array}{l}10 \mathrm{H} \\
\text { adultos }\end{array}$ & $\begin{array}{l}\text { FC } \\
\text { EC }\end{array}$ & $\begin{array}{l}33 \pm 1 \\
38 \pm 2\end{array}$ & $\begin{array}{l}0,985 \\
0,996\end{array}$ & NR & $\begin{array}{l}1,8 \\
1,8\end{array}$ & NR \\
\hline 3 & $\begin{array}{l}10 \mathrm{H} \\
\text { adultos }\end{array}$ & EJ & $53 \pm 10$ & 0,972 & NR & 3,2 & NR \\
\hline 5 & $\begin{array}{c}16 \mathrm{H} \\
\text { jovens NT }\end{array}$ & $V L$ & $23 \pm 4$ & 0,996 & 2,1 & NR & NR \\
\hline 9 & $\begin{array}{l}10 \mathrm{H} \\
\text { adultos }\end{array}$ & FP & $70 \pm 4$ & 0,962 & NR & NR & NR \\
\hline 10 & $\begin{array}{l}22 \mathrm{H} \\
\text { jovens }\end{array}$ & $\begin{array}{l}F C \\
E C \\
F J \\
E J \\
F P\end{array}$ & $\begin{array}{l}32 \pm 1 \\
41 \pm 1 \\
55 \pm 1 \\
42 \pm 1 \\
44 \pm 3\end{array}$ & NR & $\begin{array}{l}2,6 \\
1,7 \\
3,1 \\
0,9 \\
2,1\end{array}$ & NR & NR \\
\hline Fonte & $n$ & Sítio(s) & AST $\left(\mathrm{cm}^{2}\right)$ & $\mathrm{CCl}(\mathrm{R})$ & CV (\%) & $\mathrm{CR}\left(\mathrm{cm}^{2}\right)$ & $\operatorname{ETM}\left(\mathrm{cm}^{2}\right)$ \\
\hline 11 & $\begin{array}{c}38 \mathrm{M} \\
\text { pós-menopausa }\end{array}$ & $\begin{array}{l}\mathrm{RF} \\
\mathrm{BB}\end{array}$ & $\begin{array}{l}3,5 \pm 0,1 \\
4,6 \pm 0,2\end{array}$ & $\begin{array}{l}0,880 \\
0,990\end{array}$ & $\begin{array}{l}3,8 \\
3,6\end{array}$ & NR & NR \\
\hline 11 & $\begin{array}{l}85 \mathrm{He} \mathrm{M} \\
\text { idosos }\end{array}$ & RF & $\begin{array}{l}4,6 \pm 0,2(\mathrm{H}) \\
3,0 \pm 0,1(\mathrm{M})\end{array}$ & 0,720 & 3,5 & NR & NR \\
\hline 11 & $\begin{array}{l}10 \mathrm{He} \mathrm{M} \\
\text { jovens }\end{array}$ & RF & $\begin{array}{l}5,2 \pm 0,6(\mathrm{H}) \\
5,1 \pm 0,4(\mathrm{M})\end{array}$ & 0,800 & 6,7 & NR & $N R$ \\
\hline 12 & $\begin{array}{l}6 \mathrm{HeM} \\
\text { idosos }\end{array}$ & $\mathrm{VL}$ & NR & 0,997-0,999 & NR & NR & $\begin{array}{c}0,29 \\
(2,6 \%)\end{array}$ \\
\hline
\end{tabular}




\section{Imagens musculares}

As espessuras musculares de flexores e extensores do cotovelo e joelho foram medidas por meio de US bidimensional no modo $B$ (Toshiba Nemium ${ }^{\oplus}$, Japão), usando transdutor de $7,5 \mathrm{MHz}$ e gel condutor hidrossolúvel. Os sítios anatômicos identificadores dos pontos de medida foram marcados por dois antropometristas treinados (C.M.M. e P.S.C.G.), sendo sempre os mesmos nas medidas repetidas. As medidas ocorreram em dois testes intercalados por no mínimo três dias (7,3 \pm 5,2 dias), sendo todas realizadas pela mesma ultrassonografista experiente (S.P.L.), a qual, no momento da repetição das medidas, não tinha conhecimento dos valores anteriores.

Nos membros inferiores a identificação do sítio anatômico ocorreu à meia distância entre a borda superior proximal da patela e o ligamento inguinal, com o indivíduo sentado. A medida de extensores do joelho ocorreu sobre o ponto anterior da coxa direita e a de flexores, sobre o prolongamento do ponto até a face posterior da coxa esquerda.

Nos membros superiores, foi localizado o ponto médio entre a borda superior lateral do processo acromial e a fossa radial. Esse ponto foi estendido até a face anterior do braço direito, onde foi coletada a medida de flexores e até a face posterior do braço esquerdo, para a medida de extensores do cotovelo. A identificação dos sítios era registrada para reprodução na segunda visita.

Após a identificação dos sítios anatômicos, as medidas eram realizadas com o indivíduo deitado (em decúbito dorsal ou ventral) e relaxado com os membros inferiores estendidos e ligeiramente afastados e os membros superiores estendidos junto ao corpo. O transdutor era apoia- do levemente sobre a pele, perpendicularmente à superfície do membro a ser medido. Ao identificar o tecido muscular compreendido entre as interfaces com o osso e com o tecido adiposo, o ultrassonografista congelava a imagem no monitor e media a sua espessura com o cursor. O mesmo procedimento era repetido na segunda visita do sujeito. A figura 1 ilustra um exemplo típico das imagens obtidas nos testes.

\section{Análise estatística}

A normalidade da distribuição das medidas foi analisada pelo teste de Shapiro-Wilk. A associação entre as medidas repetidas foi determinada pelo coeficiente de correlação intraclasse (CCI). Uma análise de variância (ANOVA) com medidas repetidas foi empregada para identificar diferenças entre teste e reteste.

O coeficiente de variação (CV) foi estabelecido para cada sujeito a partir da divisão do desvio padrão de cada par de medidas pelos seus valores médios (CV = [([DP/média)*100]. A seguir, o CV médio foi calculado a partir da média dos CV individuais.

O limite de significância estatística foi estabelecido em $\mathrm{P}<0,05$ e, para as análises acima, utilizou-se um pacote estatístico (SPSS versão 11.0, SPSS Inc., Chicago, EUA).

O grau de concordância entre os pares de medidas obtidas interdias foi observado de acordo com o método sugerido por Bland e Altman ${ }^{(13)}$. O coeficiente de repetibilidade (CR) foi obtido através da multiplicação de 1,96 pelo desvio padrão (DP) das diferenças entre teste e reteste. O coeficiente de correlação de Pearson foi calculado entre as médias das medidas e as diferenças entre cada par de medidas para testar se os dados apresentavam erro heterocedástico.
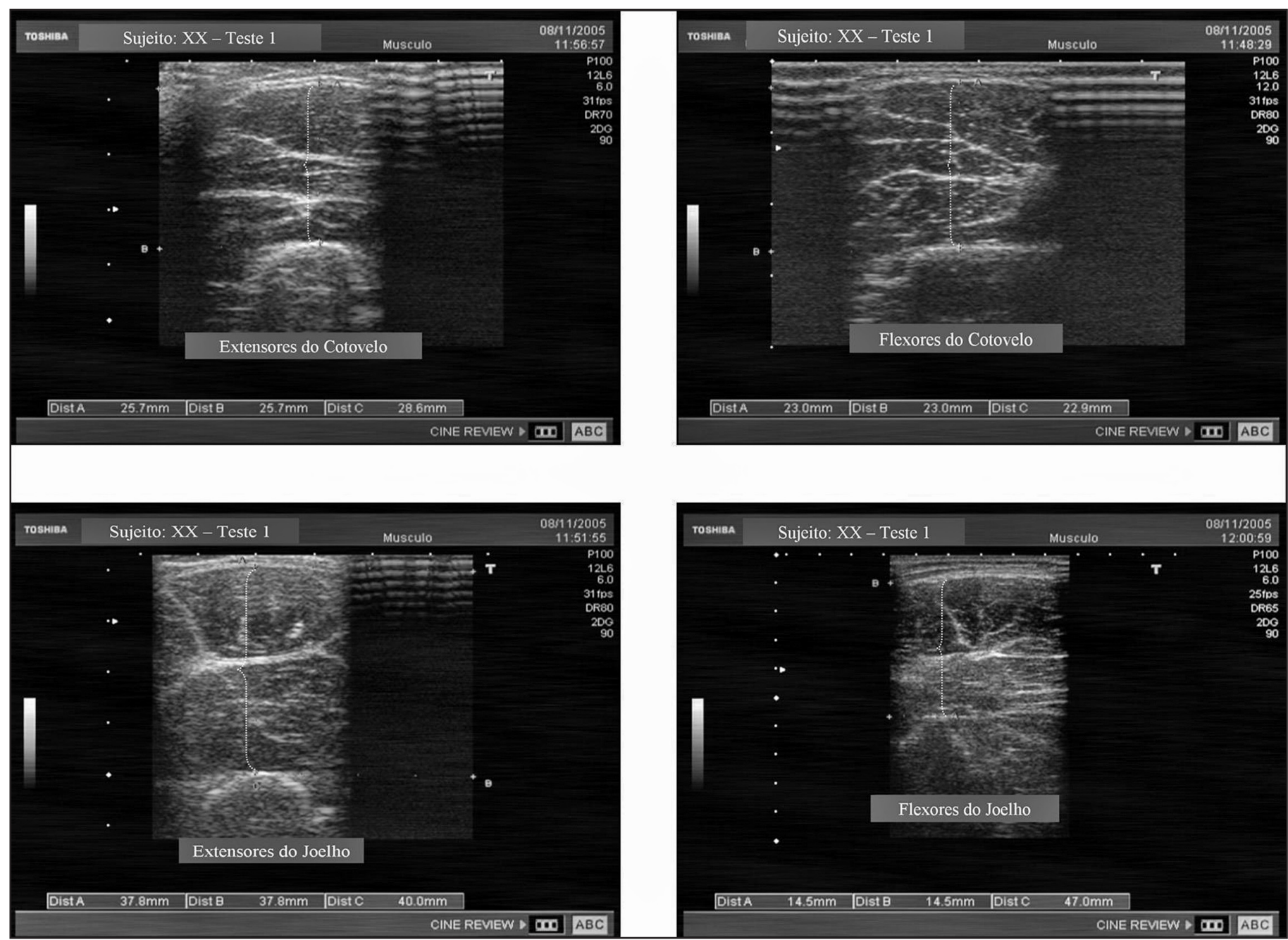

Figura 1. Exemplo típico das imagens de espessuras musculares obtidas pela ultrassonografia 
O erro típico da medida (ETM) foi considerado o resultante da razão entre o DP das diferenças obtidas entre os pares de medidas e a raiz quadrada do algarismo dois $(E T M=D P / \sqrt{2})$, conforme sugerido por Hopkins ${ }^{(14)}$.

\section{RESULTADOS}

O teste de Shapiro-Wilk apontou que os valores das medidas musculares apresentaram distribuição normal.

A ANOVA com medidas repetidas não identificou diferenças significativas entre os pares de medidas interdias em todas as variáveis observadas. Em relação ao CCl, todos alcançaram significância estatística, no entanto, o CCl das medidas de flexores do joelho foi o mais baixo, enquanto seu ETM foi o mais alto (tabela 3)

Nenhuma das variáveis apresentou coeficientes de correlação de Pearson estatisticamente significativos entre as médias dos valores obtidos nos testes e retestes e as diferenças entre cada par de medidas, indicando que não apresentaram erro heterocedástico. Para as medidas de flexores e extensores do cotovelo foram encontrados, respectivamente, $r=-0,181$ e $r=-0,198$, e flexores e extensores do joelho, $r=$ $-0,296$ e $r=-0,222(P>0,05)$.

Os gráficos de Bland-Altman demonstrando o grau de concordância entre os pares de medidas com seus respectivos CR são apresentados na figura 2. Os limites de concordância e as médias das diferenças entre testes e retestes são mostrados na tabela 4. Nas medidas de flexores do joelho observou-se que um sujeito esteve fora dos limites de concordância para cada uma das variáveis.
Tabela 3. Valores descritivos (mm), coeficiente de variação médio (CV), coeficiente de correlação intraclasse (CCl, R) e erro típico das medidas (ETM) musculares pela ultrassonografia

\begin{tabular}{|c|c|c|c|c|}
\hline Sítios & Média \pm DP & $\mathrm{CV} \%$ & $\mathrm{R}^{*}$ & ETM (\%) \\
\hline \multicolumn{5}{|c|}{ Flexores do cotovelo } \\
\hline Dia 1 & $28,6 \pm 6,9$ & \multirow{2}{*}{3,9} & \multirow{2}{*}{0,970} & \multirow{2}{*}{$1,3(4,5)$} \\
\hline Dia 2 & $28,5 \pm 7,3$ & & & \\
\hline \multicolumn{5}{|c|}{ Extensores do cotovelo } \\
\hline Dia 1 & $29,2 \pm 12,0$ & \multirow{2}{*}{6,1} & \multirow{2}{*}{0,971} & \multirow{2}{*}{$1,6(5,2)$} \\
\hline Dia 2 & $31,3 \pm 12,4$ & & & \\
\hline \multicolumn{5}{|c|}{ Flexores do joelho } \\
\hline Dia 1 & $49,9 \pm 6,5$ & \multirow{2}{*}{6,6} & \multirow{2}{*}{0,555} & \multirow{2}{*}{$4,9(9,6)$} \\
\hline Dia 2 & $52,4 \pm 8,3$ & & & \\
\hline \multicolumn{5}{|c|}{ Extensores do joelho } \\
\hline Dia 1 & $35,9 \pm 7,6$ & \multirow{2}{*}{4,6} & \multirow{2}{*}{0,929} & \multirow{2}{*}{$1,9(5,2)$} \\
\hline Dia 2 & $37,4 \pm 8,2$ & & & \\
\hline
\end{tabular}

Tabela 4. Resultados da análise de Bland-Altman (média das diferenças teste-reteste e limites de concordância) para as medidas musculares pela ultrassonografia

\begin{tabular}{lcc}
\hline \multicolumn{1}{c}{ Sítios } & Média \pm DP das diferenças & Limites de concordância \\
\hline Flexores do cotovelo & $-0,138 \pm 0,161 \mathrm{~mm}$ & $-0,4603-0,1834 \mathrm{~mm}$ \\
Extensores do cotovelo & $-2,067 \pm 2,213 \mathrm{~mm}$ & $-6,4917-2,3584 \mathrm{~mm}$ \\
Flexores do joelho & $-2,467 \pm 6,927 \mathrm{~mm}$ & $-16,3209-11,3876 \mathrm{~mm}$ \\
Extensores do joelho & $-1,447 \pm 2,712 \mathrm{~mm}$ & $-6,8709-3,9775 \mathrm{~mm}$ \\
\hline
\end{tabular}
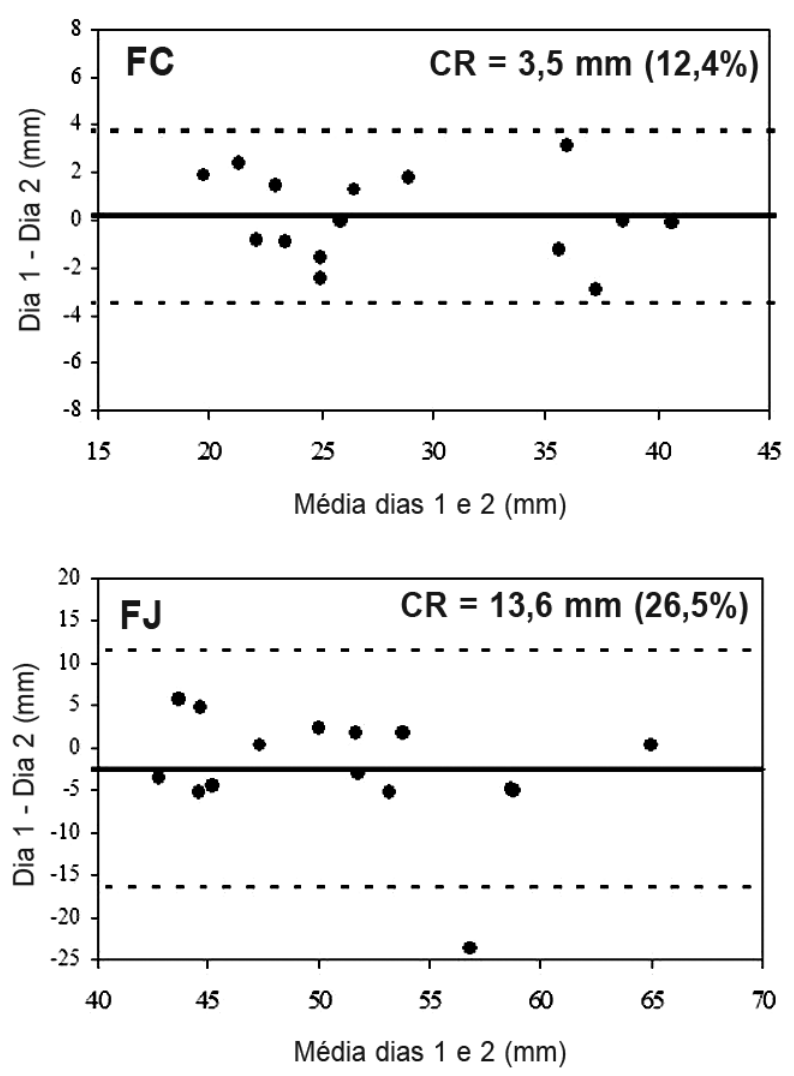
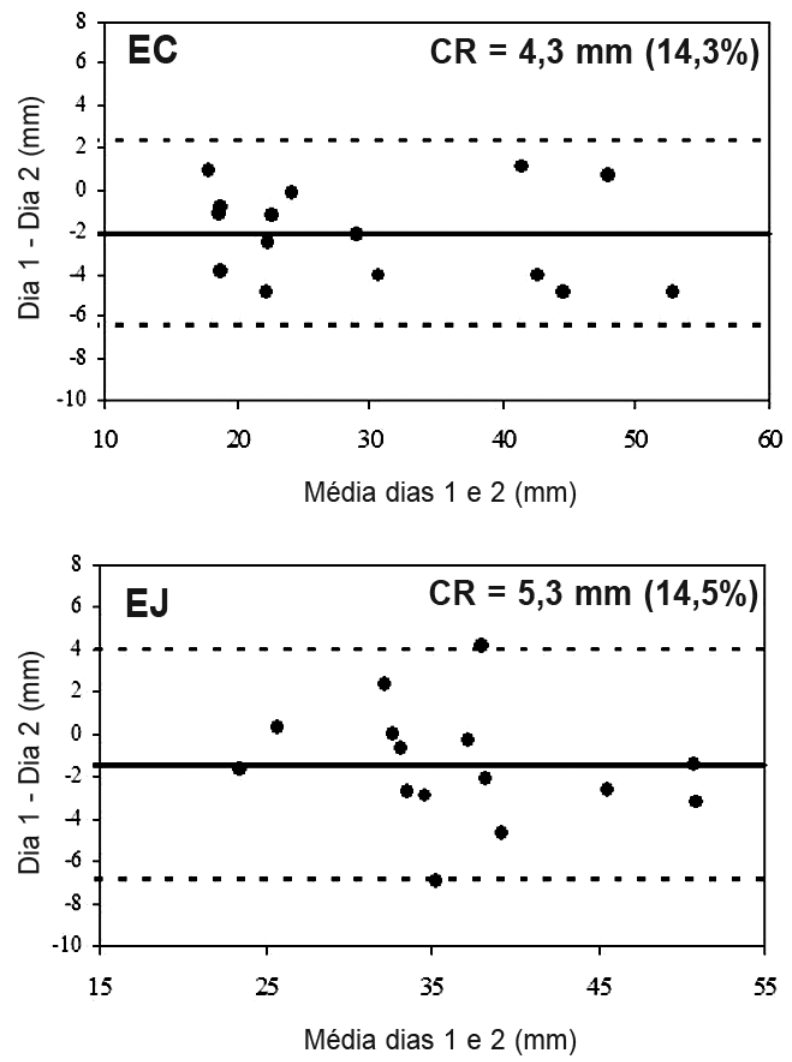

Figura 2. Análise de Bland-Altman entre as diferenças absolutas e as médias das diferenças entre teste e reteste para as variáveis estudadas, FC: flexores do cotovelo, EC: extensores do cotovelo, FJ: flexores do joelho, EJ: extensores do joelho, CR = coeficiente de repetibilidade (1,96 x desvio padrão das diferenças entre as medidas). 


\section{DISCUSSÃO}

Este estudo, desenvolvido para determinar a confiabilidade da medida de espessuras musculares pela US, encontrou medidas similares àquelas observadas anteriormente em adultos não atletas ${ }^{(2,3,8,10)}$.

Outros estudos utilizaram técnica similar à do presente e observaram coeficientes de correlação intraclasse para flexores e extensores do cotovelo e extensores do joelho semelhantes aos calculados no presente estudo ${ }^{(2,3,10)}$. Entretanto, os coeficientes de variação dos estudos anteriores foram sistematicamente menores do que os calculados no presente.

No presente estudo, as medidas de flexores do joelho apresentaram $C V=6,6 \%$ e $R=0,555$, portanto, foram consideradas não confiáveis. Candow e Chilibeck ${ }^{(10)}$, ao estudar a estabilidade das medidas de espessuras de flexores do joelho, também detectaram para essas os maiores CV (3,1\% em jovens e 3,6\% em idosos) entre todas as medidas realizadas (flexores e extensores do cotovelo e joelho e flexores plantares); contudo, tais CV foram menores do que o observado no presente estudo.

Ishida et al ${ }^{(8)}$ utilizaram técnica similar à do atual estudo, com exceção da posição dos sujeitos, que se mantinham de pé durante a aferição. Foram medidas as espessuras de 14 sítios musculares utilizando a teoria da generabilidade para a determinação da confiabilidade. Esse método estatístico se baseia no cálculo do coeficiente de generabilidade $(G)$, o qual indica a magnitude da variabilidade da medida relativa aos sujeitos e às condições experimentais. Os valores de $\mathrm{G}$ variam de 0 a 1,0 e, quanto maior o valor encontrado, maior a variabilidade devida aos sujeitos e menor a variabilidade devida às condições experimentais. Ishida et al. ${ }^{(8)}$ apontaram $G \geq 0,92$ para todas as medidas, incluindo extensores e flexores de cotovelo e joelho, levando-os a concluir que a US se mostrou confiável para obtenção das espessuras musculares.

Ishida et al. ${ }^{(8)}$ ressaltaram que a confiabilidade pode ser ameaçada pelo grau de treinamento do ultrassonografista e que maiores níveis de adiposidade subjacente ao sítio anatômico de tomada das medidas podem aumentar a dificuldade de identificação da interface músculo-tecido adiposo e, subsequentemente, prejudicar a qualidade da medida.

Vale lembrar que as técnicas utilizadas variam de acordo com o estudo em questão e alguns deles não informaram a confiabilidade das medidas de espessuras, mas sim do volume ${ }^{(1)}$ ou da área de seção transversa ${ }^{(11)}$ de músculos específicos, dificultando a comparação com os presentes resultados.

A análise dos gráficos de Bland e Altman ${ }^{(13)}$ apontou que as variáveis não apresentaram erros heterocedástico nem sistemático absoluto, ou seja, as diferenças entre teste e reteste não guardaram relação significativa com a magnitude da medida nem apresentaram tendências a serem sistematicamente positivas ou negativas. Ressalta-se que a variabilidade na repetibilidade das medidas foi grande, uma vez que apenas a de flexores do cotovelo apresentou média das diferenças entre teste e reteste próxima de zero. Para as demais, tanto as médias quanto os limites de concordância foram altos (tabela 4 e figura 2).

Essa observação, juntamente com o conhecimento do ETM de cada sítio muscular, confirma a necessidade de considerar de relevância clínica apenas as modificações maiores do que o ETM; dessa forma, eliminase a interferência da imprecisão do avaliador quando da utilização das espessuras musculares pela US, tão importante em acompanhamentos longitudinais, seja de caráter clínico ou científico.

Entre alguns estudos ${ }^{(4-6)}$ que empregaram a US para monitorar modificações musculares com o treinamento contrarresistência, um deles ${ }^{(4)}$ utilizou como referência a espessura de grupamentos musculares compreendidos entre as interfaces com o osso e com o tecido adiposo.

Starkey et al. ${ }^{(4)}$ efetuaram medidas de espessuras de 10 sítios da musculatura da coxa do membro direito de sujeitos inicialmente destreinados. Após 14 semanas de treinamento contrarresistência realizado três vezes por semana e constando de uma ou três séries de exercícios de extensão e flexão de joelhos, foram detectadas modificações musculares estatisticamente significativas para ambos os grupos. Entretanto, algumas dessas foram de magnitudes bastante modestas, como, por exemplo, a ocorrida na face ântero-medial da coxa, que aumentou de $38,8 \pm 7,2 \mathrm{~mm}$ para $39,8 \pm 6,5 \mathrm{~mm}$. Esse estudo não cita o ETM da medida, considerando apenas o teste estatístico para estabelecer o efeito do treinamento sobre as modificações nas dimensões musculares.

Aparentemente, não há resultados referentes ao ETM de medidas de espessuras musculares provenientes de estudos que utilizaram métodos semelhantes aos aqui apresentados. Neste, a medida da espessura de flexores do joelho apresentou ETM de $4,9 \mathrm{~mm}$, o que desaconselha sua utilização para fins de monitoramento de modificações musculares, principalmente quando são esperadas pequenas alterações.

Em conclusão, os resultados aqui descritos demonstraram que, à exceção de flexores do joelho, as medidas de espessuras musculares pela US apresentaram alta confiabilidade, o que permite seu uso para fins de monitoramento das modificações musculares devidas a intervenções como programas de dieta, treinamento físico ou reabilitação.

Todos os autores declararam não haver qualquer potencial conflito de interesses referente a este artigo.

\section{REFERÊNCIAS BIBLIOGRÁFICAS}

1. Esformes Jl, Narici MV, Maganaris CN. Measurement of human muscle volume using ultrasonography Eur J Appl Physiol 2002;87:90-2.

2. Miyatani M, Kanehisa H, Fukunaga T. Validity of bioelectrical impedance and ultrasonographic methods for estimating the muscle volume of the upper arm. Eur J Appl Physiol 2000;82:391-6.

3. Miyatani M, Kanehisa H, Kuno S, Nishijima T, Fukunaga T.Validity of ultrassonograph muscle thickness measurements for estimating muscle volume of knee extensors in humans. Eur J Appl Physiol 2002;86:203-8.

4. Starkey DB, Pollock ML, Ishida Y, Welsch MA, Brechue WF, Graves JE, et al. Effect of resistance training volume on strength and muscle thickness. Med Sci Sports Exerc 1996;28:1311-20.

5. Alegre LM, Jiménez F, Gonzalo-Orden J, Martín-Acero R, Aguado X. Effects of dynamic resistance training on fascicle length and isometric strength. J Sports Sci 2006;24:1-8.

6. Blazevich AJ, Gill ND, Deans N, Zhou S. Lack of human muscle architectural adaptation after short-term strength training. Muscle Nerve 2007;35:78-86.

7. Gondin J, Guette M, Ballay Y, Martin A. Neural and muscular changes to detraining after electrostimulation training. Eur J Appl Physiol 2006;97:165-73.
8. Ishida Y, Carroll JF, Pollock ML, Graves JE, Leggett SH. Reliability of B-mode ultrasound for the measurement of body fat and muscle thickness. Am J Hum Biol 1992;4:511-20.

9. Miyatani M, Kanehisa H, Ito M, KawakamiY, Fukunaga T. The accuracy of volume estimates using ultrasound muscle thickness measurements in different muscle groups. Eur J Appl Physiol 2004;91:264-72.

10. Candow DG, Chilibeck PD. Differences in size, strength, and power of upper and lower body muscle groups in young and older men. J Gerontol Bio Sci Med Sci 2005;60:148-56.

11. Bemben MG. Use of diagnostic ultrasound for assessing muscle size. J Strength Cond Res 2002;16:103-8.

12. Reeves ND, Maganaris CN, Narici MV. Ultrasonographic assessment of human skeletal muscle size. Eur J Appl Physiol 2004;91:116-8.

13. Bland JM, Altman DG. Statistical methods for assessing agreement between two methods of clinical measurement. Lancet 1986;8:307-10.

14. Hopkins WG. Measures of reliability in sports medicine and science. Sports Med 2000;30:1-15. 